\title{
STOCHASTIC COMPARISON OF DISCOUNTED REWARDS
}

\author{
RHONDA RIGHTER, ${ }^{*}$ University of California
}

\begin{abstract}
It is well know that the expected exponentially discounted total reward for a stochastic process can also be defined as the expected total undiscounted reward earned before an independent exponential stopping time (let us call this the stopped reward). Feinberg and Fei (2009) recently showed that the variance of the discounted reward is smaller than the variance of the stopped reward. We strengthen this result to show that the discounted reward is smaller than the stopped reward in the convex ordering sense.
\end{abstract}

Keywords: Total discounted reward; stopping time; stochastic ordering

2010 Mathematics Subject Classification: Primary 60G40

Secondary $90 \mathrm{C} 40$

Consider the following definitions of Feinberg and Fei (2009). Let

$$
J_{1}=\int_{0}^{\infty} \mathrm{e}^{-\alpha t} r_{t} \mathrm{~d} t+\sum_{n=1}^{\infty} \mathrm{e}^{-\alpha T_{n}} R_{n},
$$

where $r_{t}$ is an $\mathcal{F}_{t}$-adapted stochastic process representing the reward rate at time $t, \mathcal{F}_{t}$ is an increasing filtration on a probability space $(\Omega, \mathcal{F}, \mathrm{P}), T_{n}$ is an $\mathcal{F}_{t}$-adapted stopping time, and $R_{n}$ is an $\mathcal{F}_{T_{n}}$-adapted stochastic sequence representing lump sum rewards, and let

$$
J_{2}=\int_{0}^{T} r_{t} \mathrm{~d} t+\sum_{n=1}^{N(T)} R_{n},
$$

where $T$ has an exponential distribution with rate $\alpha$, independent of $\mathcal{F}_{\infty}$, and

$$
N(t)=\sup \left\{n: T_{n} \leq t\right\}
$$

is the number of stopping times before $T$. Then $J_{1}$ represents the total discounted reward and $J_{2}$ represents the total undiscounted reward earned before an exponential time $T$. It is well known that $\mathrm{E} J_{1}=\mathrm{E} J_{2}$, and Feinberg and Fei (2009) showed that $\operatorname{var}\left(J_{1}\right) \leq \operatorname{var}\left(J_{2}\right)$. We show the following more general result, where, for two random variables $X$ and $Y, X \leq_{\mathrm{cx}} Y$ if and only if $\mathrm{E} f(X) \leq \mathrm{E} f(Y)$ for all convex functions $f$.

Theorem 1. It holds that $J_{2} \geq_{\mathrm{cx}} J_{1}$.

Proof. Let us make the dependency on $T$ explicit, and write $J_{2}(T)$. We have

$$
\mathrm{E}\left[J_{2}(T) \mid \mathcal{F}_{\infty}\right]=J_{1} \mid \mathcal{F}_{\infty},
$$

so $J_{2}(T)\left|\mathcal{F}_{\infty} \geq_{\mathrm{cx}} J_{1}\right| \mathcal{F}_{\infty}$, because, from Jensen's inequality, for any random variable $X$,

Received 19 July 2010; revision received 10 November 2010.

* Postal address: Department of Industrial Engineering and Operations Research, University of California, Berkeley, CA 94720, USA. Email address: rrighter@ieor.berkeley.edu 
$X \geq_{\mathrm{cx}} \mathrm{E} X$. Therefore, for any convex function $f$,

$$
\mathrm{E}\left[f\left(J_{2}(T)\right) \mid \mathcal{F}_{\infty}\right] \geq_{\mathrm{cx}} \mathrm{E}\left[f\left(J_{1}\right) \mid \mathcal{F}_{\infty}\right],
$$

so

$$
\mathrm{E}\left[f\left(J_{2}(Y)\right)\right]=\mathrm{E}\left[\mathrm{E}\left[f\left(J_{2}(T)\right) \mid \mathcal{F}_{\infty}\right]\right] \geq_{\mathrm{cx}} \mathrm{E}\left[f\left(J_{1}\right) \mid \mathcal{F}_{\infty}\right]=\mathrm{E}\left[f\left(J_{1}\right)\right],
$$

and, therefore, $J_{2}(T) \geq_{\mathrm{cx}} J_{1}$.

Note that the argument goes through with arbitrary discounting, not necessarily exponential discounting. That is, if the reward rate at time $t$ is $\alpha(t)$, where $\alpha(0)=1$ and $\lim _{t \rightarrow \infty} \alpha(t)=0$, so

$$
J_{1}=\int_{0}^{\infty} \alpha(t) r_{t} \mathrm{~d} t+\sum_{n=1}^{\infty} \alpha\left(T_{n}\right) R_{n},
$$

then, again, $J_{2}(T) \geq_{\mathrm{cx}} J_{1}$, where $J_{2}(T)$ is as defined above with $T$ having tail distribution $\alpha$ : $\alpha(t)=\mathrm{P}\{T>t\}$.

\section{Reference}

FeInBERG, E. A. AND FeI, J. (2009). An inequality for variances of the discounted rewards. J. Appl. Prob. 46, 1209-1212. 